\title{
Nuevas aportaciones a la flora liquénica de la Comunitat Valenciana (E de España)
}

\section{S. FOS MARTÍN}

\author{
VAERSA-Generalitat Valenciana, av. Corts Valencianes, 20, ES-46015 Valencia, España
}

ORCID iD. S. FOS MARTÍN: https://orcid.org/0000-0003-0976-5459

E-mail: flora_catalogada@gva.es

Editor: L. Sáez

Recibido 19 julio 2018; aceptado 3 octubre 2018; publicado on line 17 julio 2019

\begin{abstract}
New contributions to the lichen flora of the Valencian Community (E of Spain). - Taxonomic, ecological and chorological data are provided on 20 new taxa for the lichen flora of the Valencian Community. Many contributions can be also considered significant for its rarity at the peninsular level (Calicium notarisii, Caloplaca nana, Dirina fallax, Lecania sambucina, Lecanora rupicola subsp. subplanata, Myriolecis reuteri, Rhizocarpon umbilicatum, Staurothele hymenogonia, Xanthoria aureola) or in the Iberian-Mediterranean areas (Bryoria capillaris, Hydropunctaria maura, Ochrolechia alboflavescens, Verrucaria halizoa). Candelariella commutata and Catinaria neuschildii are found for the first time in the Iberian Peninsula.
\end{abstract}

Key words: distribution; floristic novelties; Iberian Peninsula; lichens.

\section{Resumen}

NueVas aportaciones a la flora liquénica de la Comunitat Valenciana (E de España). - Se aportan datos taxonómicos, ecológicos y corológicos sobre 20 nuevos táxones para la flora liquénica de la Comunitat Valenciana. Muchas aportaciones también resultan significativas por su rareza a e scala peninsular (Calicium notarisii, Caloplaca nana, Candelariella commutata, Dirina fallax, Lecania sambucina, Lecanora rupicola subsp. subplanata, Myriolecis reuteri, Rhizocarpon umbilicatum, Staurothele hymenogonia, Xanthoria aureola) o en los territorios mediterráneos ibéricos (Bryoria capillaris, Hydropunctaria maura, Ochrolechia alboflavescens, Verrucaria halizoa), destacando especialmente Candelariella commutata y Catinaria neuschildii que se citan por primera vez en la Península Ibérica.

Palabras clave: corología; líquenes; novedades florísticas; Península Ibérica.

\section{Cómo citar este artículo / Citation}

Fos Martín, S. 2019. Nuevas aportaciones a la flora liquénica de la Comunitat Valenciana (E de España). Collectanea Botanica 38: e006. https://doi.org/10.3989/collectbot.2019.v38.006

\section{Copyright}

(C) 2019 CSIC. This is an open-access article distributed under the terms of the Creative Commons Attribution 4.0 International (CC BY 4.0) License. 


\section{INTRODUCCIÓN}

La primera aproximación a la Check-List de los líquenes de la Comunitat Valenciana estableció un catálogo de 569 táxones infragenéricos basado exclusivamente en fuentes bibliográficas que proporcionaron más de 3700 citas (Atienza \& Segarra, 1999a). En la actualidad, la Lista Patrón de líquenes y hongos liquenícolas del Banco de Datos de Biodiversidad de la Comunitat Valenciana (BDBCV; http://bdb.cma.gva.es) alcanza un valor de 909 táxones infragenéricos: 822 hongos liquenizados y 87 liquenícolas (BDBCV, 2017). Este notable incremento es el resultado de las aportaciones publicadas en este dilatado periodo en trabajos florísticos (Alonso \& Egea, 1999; Atienza et al., 2001; Fos, 2001; Fos et al., 2001; Fos \& Barreno, 2002; Atienza \& Fos, 2011; Garrido-Benavent et al., 2013; Boom \& Etayo, 2014), en revisiones taxonómicas que han incluido material valenciano (Calatayud et al., 2002, 2013; Giralt \& Nordin, 2002; Atienza et al., 2003; Boqueras \& Llimona, 2003; Llop, 2003; Rico et al., 2003; Martínez \& Burgaz, 2008; Gaya, 2009; Giralt et al., 2009; Navarro-Rosinés et al., 2009; Carvalho, 2009, 2012; Prieto et al., 2010a, b; Burgaz, 2011, 2015a; Pino-Bodas et al., 2012, 2013; Carballal, 2013; Tehler et al., 2013) y a las novedades florísticas territoriales (Gaya \& Pérez, 2002; Crespo et al., 2004; Fos et al., 2017) o para la ciencia (Calatayud \& Etayo, 2001; Calatayud \& Navarro-Rosinés, 2001; Calatayud \& Triebel, 2001, 2003; Atienza, 2002; Calatayud \& Barreno, 2003; Divakar et al., 2005; Fos \& Giralt, 2009; Muñiz et al., 2009; Llop, 2010; Lumbsch et al., 2011; Molina et al., 2011; Garrido-Benavent \& Pérez-Ortega, 2015). La intensa labor de prospección desarrolla$\mathrm{da}$, especialmente en la última década, constituye la otra fuente de información que ha conducido al conocimiento actual de la biodiversidad liquénica regional. Toda la información florística generada con esta actividad ha sido introducida en el BDB$\mathrm{CV}$ y se encuentra disponible para su consulta virtual. A finales de 2017, las referencias a líquenes $\mathrm{y}$ hongos liquenizados acumuladas en esta base de datos ascendían a un total de 44.228 citas georreferenciadas (BDBCV, 2017).

El objetivo de este trabajo es contribuir al conocimiento de la flora liquénica de la Comunitat Valenciana, presentando las novedades florísticas más significativas que se han venido encontrando en diferentes campañas realizadas en los últimos años. Este trabajo pretende ser el primero de una serie destinada a mostrar los avances florísticos, biogeográficos y de conservación que se vienen realizando en este territorio.

\section{MATERIAL Y MÉTODOS}

La metodología ha sido la habitual en los trabajos de liquenología. El material fue identificado en el laboratorio con ayuda de las floras liquénicas generales, haciendo uso de monografías sobre algunos géneros y especies concretos que son referenciadas en cada caso y recogidas en la bibliografía. El listado de especies ha sido confeccionado a partir del material recolectado en diferentes campañas de muestreo. Para la nomenclatura y autoría de los táxones se sigue el Index Fungorum (http://www. indexfungorum.org) y a Nimis (2016) para los táxones no confirmados por el primero. Los ejemplares han sido depositados en la sección de líquenes del Herbario de la Facultat de Ciències Biològiques de la Universitat de València (VAL Lich.). Los táxones han sido ordenados alfabéticamente, indicando para cada cita la localidad de recolección, la cuadrícula UTM $1 \times 1 \mathrm{~km}$ (Datum ETRS89), la altitud, el sustrato, la fecha de recolección, el recolector o recolectores y el número de pliego. Para los táxones recientemente recombinados, se indican los sinónimos previos, cuyo uso se encuentra bastante más extendido. Toda la información se encuentra disponible para su consulta en el BDBCV (http://bdb. cma.gva.es) y en la plataforma de datos de biodiversidad GBIF (https://www.gbif.org). Estas bases de datos incluyen otras observaciones (visto vivo) de las especies incluidas en este trabajo. Además, la primera también permite consultar el conjunto de especies identificadas en la misma localidad.

\section{RESULTADOS}

Bryoria capillaris (Ach.) Brodo \& D. Hawksw.

Epífito, preferentemente sobre coníferas, aunque también en planifolios, la encontramos enmarañada entre las ramificaciones terminales más o menos defoliadas de Juniperus communis subsp. hemisphaerica (C. Presl) Nyman y sobre las ramas bajas y más o menos expuestas de Pinus sylvestris 
L., en un pinar supramediterráneo (Sabino-Pinetum sylvestris Rivas Goday \& Borja 1961) bien estructurado y con ejemplares monumentales. El paraje está incluido en la Microrreseva de Flora (MRF) "Pino de Vicente Tortajada", disfrutando de la protección territorial asociada a esta figura (Laguna et al., 2016). Su presencia en estos puntos concuerda con las observaciones de Etayo (1989) que la encuentra confinada en bosques viejos y poco alterados de Navarra.

Las aproximaciones filogenéticas más recientes de la sección Implexae del género Bryoria Brodo $\&$ D. Hawksw., basadas en datos moleculares y en algunos caracteres químicos y morfológicos, confirmaron la necesidad de revisar la delimitación específica de algunos táxones. Diversas especies y muestras, entre las que se incluye $B$. capillaris, eran excepciones al marcado carácter monofilético que caracteriza a la mayoría de sus congéneres. En esta especie, los ejemplares analizados se separan en dos subclados que responden a su distribución geográfica (Norteamérica vs. Europa y Asia) y, parcialmente, a los metabolitos secundarios (Myllys et al., 2011, 2014). Para resolver este conflicto se propone el tratamiento de los ejemplares norteamericanos de $B$. capillaris como conespecíficos de B. pikei Brodo \& D. Hawksw. y se restringe el binomen Bryoria capilaris s. str. al material euroasiático, que queda constituido como un grupo monofilético basado en datos moleculares y caracteres morfológicos (Velmala et al., 2014).

Su área de distribución se extiende por la mayor parte de Europa, hasta la Región Mediterránea, y también está presente en Asia boreal y en las zonas más elevadas de Irán, las Islas Azores y las Canarias (Hafellner, 1995; Velmala et al., 2014). En la Península Ibérica, su óptimo se localiza en los bosques de coníferas maduros y estables de la Región Eurosiberiana. En los territorios mediterráneos, aparece de forma más rara y dispersa, restringida a los bosques de coníferas supra y oromediterráneos de los principales sistemas montañosos (Central, Ibérico y Bético), normalmente en áreas con frecuentes vientos húmedos y fríos (Aragón, 2002; Aragón et al., 1999, 2001, 2006; Calatayud \& Sanz, 2000; Burgaz et al., 2017).

España, Valencia: Puebla de San Miguel, Sierra de Javalambre, MRF "Pino de Vicente Tortajada", sobre Pinus sylvestris, 30SXK615374, 1700 m, 29.VIII.2006, Fos (VAL_Lich. 28064); ibid., sobre
Juniperus hemisphaerica, 7.IX.2005, Fos (VAL Lich. 28099).

Calicium notarisii (Tul.) M. Prieto \& Wedin

$=$ Cyphelium notarisii (Tul.) Blomb. \& Forssell

Preferentemente lignícola, sobre madera seca, alterada y expuesta, en tocones y troncos decorticados, también sobre postes telefónicos y vallas viejas. Coloniza con menor frecuencia la corteza de viejos árboles de corteza ácida, principalmente coníferas (Pinus pinaster Ait., P. sylvestris), en hábitats abiertos y bien iluminados.

Calicium notarisii se encuentra ampliamente distribuido en las áreas templadas del Hemisferio Norte, aunque en la Península Ibérica resulta bastante raro, con citas dispersas en Madrid, Valladolid, Soria, Teruel, Valencia y Castellón, siempre en localidades continentales en un rango altitudinal entre los 900 y los $1400 \mathrm{~m}$ de altitud (Sarrión et al., 1999; Hawksworth, 2004; Pérez-Ortega, 2007; Atienza et al., 2009; Etayo, 2010; Muñiz \& Hladun, 2011).

España, Castellón: El Toro, Sierra de Javalambre, Collado de los Perros, sobre madera alterada de Juniperus, 30SXK876181, 1560 m, 25.VII.2013, Fos (VAL_Lich. 30452).

\section{Caloplaca nana (Gaya) Nav.-Ros. \& Cl. Roux}

Saxícola calcícola, crece en paredes verticales y extraplomos de orientación norte, en posiciones protegidas de la escorrentía del agua de lluvia y de la insolación directa, normalmente acompañada por especies de preferencias nitrófilas: Calogaya arnoldii (Wedd.) Arup, Frödén \& Søchting, C. pusilla (A. Massal.) Arup, Frödén \& Søchting y Variospora flavescens (Huds.) Arup, Frödén \& Søchting (Gaya, 2009). Especie diagnóstica de $\mathrm{Ve}$ rrucarietea nigrescentis V. Wirth 1980, se incluye entre las características de Caloplacetum arnoldii Clauzade et Roux 1975, comunidad calcícola, más o menos basófila, fotófila, pero no o muy raramente heliófila, marcadamente ombrófoba y nitrófila, con óptimo en el supramediterráneo y en el montano inferior (Roux et al., 2009). En las Hoces del Cabriel (Valencia), aparece en paredes verticales orientadas al noroeste, formando parte de comunidades que se ajustan a las características indicadas y acompañada por V. flavescens, Verrucaria nigrescens Pers., Rinodina immersa (Körb.) J. Steiner, Sarcogyne regularis Körb. y Flavoplaca granulosa (Müll. 
Arg.) Arup, Frödén \& Søchting. Sin embargo, se observan diferencias en cuanto a su posición bioclimática, puesto que todo este tramo del río Cabriel corresponde al piso mesomediterráneo, localmente atemperado por la influencia mediterránea que recibe a través de la cuenca fluvial.

Su distribución está mal conocida, probablemente porque pasa fácilmente inadvertida debido a sus reducidas dimensiones. Las referencias previas la sitúan en Austria, Francia y España, donde sólo se conoce en un reducido número de localidades catalanas (Gaya, 2009; Roux et al., 2009).

España, Valencia: Venta del Moro, Parque Natural (PN) de las Hoces del Cabriel, Hoz del Rabo de la Sartén, sobre paredes calizas verticales, 30SXJ287724, 610 m, 19.V.2010, S. Fos (VAL Lich. 27306, 27314).

\section{Candelariella commutata Otte \& M. Westb.}

Recientemente descrita para establecer la identidad de los ejemplares referidos a Candelariella unilocularis (Elenkin) Nimis (Otte et al., 2013), se caracteriza por sus grandes apotecios (hasta 2 $\mathrm{mm}$ de diámetro), provistos de un reborde talino grueso y persistente, y por sus largas esporas (20$28 \mu \mathrm{m})$ con forma de salchicha. Coloniza restos vegetales, musgos epilíticos y suelos calcáreos, con frecuencia como comófito de calizas y dolomías. Los ejemplares estudiados mostraron esta ecología, ocupando las grietas y anfractuosidades de las rocas calizas planas o poco inclinadas que afloran superficialmente en los pastizales vivaces supra y oromediterráneos, en los claros del pinar albar con sabina rastrera (Sabino-Pinetum sylvestris) en la Sierra de Javalambre (Puebla de San Miguel, Valencia), o de pinares negrales [Pinus nigra subsp. salzmanii (Dunal) Franco] de repoblación en zonas menos elevadas en Portell de Morella (Castellón).

Se trata de una especie ártico-alpina muy extendida por las altas montañas de la zona holártica meridional (Nimis, 2016). Conocida del Cáucaso, los Alpes y las montañas de centro de Italia, el norte de Escandinavia y en el archipiélago de Nueva Zembla-Rusia septentrional-(Otte et al., 2013; Nimis, 2016). No se conocen citas previas para la Península Ibérica, aunque es muy probable que la referencia a $C$. aurella var. unilocularis (Elenkin) Zahlbr. de Palencia (López de Silanes et al., 1998) corresponda a esta especie.
España, Castellón: Portell de Morella, Barranc del Maset de les Pataquetes, en grietas de la roca caliza, 30TYK322970, 950 m, 6.V.2015, Fos \& Gómez-Serrano (VAL Lich. 31637). Valencia: Puebla de San Miguel, PN de Puebla de San Miguel, Sierra de Javalambre, La Hoz, comófito en roca caliza, 30TXK611355, 1525 m, 24.VI.2015. Fos, Oltra \& Gómez-Serrano (VAL_Lich. 31259, 31261).

\section{Catinaria neuschildii (Körb.) P. James}

= Catillaria neuschildii (Körb.) Th. Fr.

Se trata de una especie cortícola y lignícola poco conocida, muy próxima a $C$. atropurpurea (Schaer.) Vězda \& Poelt, pero con 12-16 esporas por asco. Coloniza los troncos de árboles viejos, tanto planifolios como coníferas, en bosques montanos y boreales (Boom \& Palice, 2006; Smith et al., 2009; Nimis, 2016). Muestra preferencia por posiciones poco afectadas por la lluvia, como la parte inferior de las ramas gruesas. El material estudiado ha sido recolectado sobre sabina albar (Juniperus thurifera L.) en los sabinares supramediterráneos continentales (Junipero hemisphaerico-thuriferae Rivas-Martínez 1969) de las montañas del interior de Castellón, a unos $1500 \mathrm{~m}$ de altitud, acompañada por Lecidella elaeochroma (Ach.) M. Choisy, Rinodina mayrhoferi A. Crespo, Physconia perisidiosa (Erichsen) Moberg y Megaspora verrucosa var. mutabilis (Ach.) Nimis $\&$ Cl. Roux, entre otras.

Se conoce en el oeste de Norteamérica, Europa central y septentrional y Siberia (Santesson et al., 2004; Boom \& Palice, 2006; Liška et al., 2008; Spribille et al., 2008; Zhdanov, 2010; Tarasova et al., 2017; Nimis et al., 2018). Extremadamente rara en toda su área de distribución, ha sido clasificada como "Vulnerable" en las Islas Británicas (Woods \& Coppins, 2012). Por esta razón, se incluye entre las especies que incrementan el interés general de conservación de los bosques caducifolios en Gran Bretaña, evaluados mediante un nuevo Índice de Continuidad Ecológica (Coppins \& Coppins, 2002). También aparece en la Lista Roja italiana de líquenes epífitos con la calificación de "Datos insuficientes" (Nascimbene et al., 2013). No se conocen citas previas para la Península Ibérica. Las referencias que muestra GBIF para Cádiz corresponden realmente a Catillaria praedicta Tretiach \& Hafellner (Fos, 1998; Tretiach \& Hafellner, 1998). 
España, Castellón: El Toro, Sierra del Toro, Barranco de la Umbría, sobre Juniperus thurifera, 30SXK874193, 1470 m, 3.V.1995, Fos (VAL_Lich. 28079).

\section{Dermatocarpon miniatum (L.) W. Mann}

Saxícola, crece en rocas calcáreas y silíceas básicas, desde esquistos calcáreos hasta calizas y dolomías, en superficies fuertemente inclinadas o extraplomadas, en hábitats húmedos o en posiciones afectadas por la escorrentía durante los episodios de lluvia. Su destacable plasticidad ha estimulado la descripción de numerosas variedades basadas en caracteres morfológicos (Clauzade \& Roux, 1985; Heiömarsson, 2001), aunque, en su mayoría, carecen de valor taxonómico en la actualidad. Aparece en un amplio rango altitudinal que se extiende desde el nivel del mar hasta el piso alpino (Egea \& Rowe, 1987; Renobales, 1996; Nimis, 2016), aunque en la Comunitat Valenciana sólo ha sido encontrada en áreas interiores meso y supramediterráneas. Subcosmopolita (Egea \& Rowe, 1987), se encuentra ampliamente distribuida en Europa y en España (Hladun \& Llimona, 2002-2014).

España, Castellón: Vistabella del Maestrat, Macizo del Penyagolosa, Barranc de l'Avellanar, 30TYK261589, 1255 m, sobre roca caliza, 5.VI.2007, Fos (VAL_Lich. 27193); Morella, Muntanyes de Vallivana, Barranc del Tell, comófito en grietas de la roca caliza, 31TBE475954, 985 m, 8.IX.2016, Fos, Gómez-Serrano, Agueras \& Sanchis (VAL_Lich. 31142).

\section{Diploschistes candidissimus (Kremp.) Zahlbr.}

Saxícola de rocas calcáreas compactas perteneciente al grupo de Diploschistes actinostomus (Ach.) Zahlbr., constituido por varias microespecies muy próximas y no siempre fáciles de separar, que se caracterizan por sus apotecios con disco punctiforme. De hecho, Etayo (2010) indica que algunas de sus muestras atribuidas a D. actinostomus pueden corresponder al críptico D. candidissimus. Se reconoce por su talo de color blanco fuertemente pruinoso y por sus preferencias calcícolas. El material estudiado ha sido herborizado sobre suelo arcilloso con abundantes guijarros en una localidad cercana a la costa, formando parte de costras liquénicas con Fulgensia fulgens (Sw.) Elenkin, Diploschistes diacapsis (Ach.) Lumbsch y Psora decipiens (Hedw.) Hoffm., entre otras.
Especie circunmediterránea principalmente distribuida por el sur de Europa, el norte de África y Asia occidental; también está presente en áreas de clima mediterráneo del sur de Australia y en la India. Su presencia en estas zonas ha llevado a proponer una distribución cosmopolita para esta especie (Lumbsch, 1989). Bastante rara en España, sólo ha sido citada en Castilla y León (Pérez-Ortega, 2007), Madrid [Barreno \& Merino, 1981 sub Diploschistes calcareus (Müll. Arg.) J. Steiner], Navarra (Etayo \& Breuss, 1996), Cataluña, Aragón (Lumbsch, 1989) y Murcia (Navarro-Rosinés \& Hafellner, 1996; Egea \& Llimona, 1997).

España, Castellón: Alcalá de Xivert, Playa del Serradal, sobre suelo pedregoso, 31TBE674554, 2 m, 7.IV.2014, Fos (VAL_Lich. 30383).

\section{Dirina fallax De Not.}

Muy similar a Dirina massiliensis Durieu \& Mont., con la que está estrechamente relacionada. Se diferencia por su talo más delgado, de color marrón oscuro hasta grisáceo o blanco cremoso, que contrasta con el blanco de $D$. massiliensis. También difieren en sus preferencias ecológicas: D. fallax únicamente coloniza rocas silíceas y ácidas, mientras que $D$. massiliensis está estrictamente confinada a las rocas calcáreas. Ambas especies incluyen especímenes sorediados que resultan morfológicamente indistinguibles (Tehler et al., 2013). Los ejemplares recolectados crecían sobre rocas subvolcánicas básicas (ofitas) en acantilados costeros de orientación norte, en comunidades dominadas por Diploicia subcanescens (Werner) Hafellner \& Poelt y Roccella phycopsis Ach., donde resultan puntualmente abundantes algunas especies de preferencias nitrófilas, como Xanthoria calcicola Oxner, Caloplaca littorea Tav. y C. marina (Wedd.) Zahlbr.

Su área de distribución se extiende desde el occidente de la Región Mediterránea hasta las costas atlánticas de Europa y África, desde Suecia hasta Marruecos y las Islas Canarias (Tehler et al., 2013; Svensson et al., 2017). El material recolectado por los autores de la reciente monografía en Almería (Cabo de Gata), Málaga (Punta de la Chullera) y Cádiz (Embalse del Celemín) son las únicas citas conocidas para la Península Ibérica, aunque es probable que las referencias ibéricas a $D$. paradoxa subsp. africana (Fée) Tehler, localizadas precisamente en el mismo Cabo de Gata (Egea \& Llimona, 1984, 
1994; Egea, 1989) y en las Islas Columbretes (Castellón) (Calatayud, 1998; Atienza \& Segarra, 1999b) correspondan a esta especie, ya que $D$. paradoxa (Fée) Tehler es una especie caribeña ausente en el Mediterráneo (A. Tehler, com. pers.) y la subespecie africana ha sido incluida como sinónimo de $D$. monothalamia Tehler \& Ertz, especie exclusiva de Cabo Verde y de Senegal (Tehler et al., 2013). Las características ecológicas y morfológicas apoyarían esta indicación, pero es necesario revisar el material de herbario para confirmar esta suposición.

España, Alicante: Alicante, Isla de Tabarca, La Mina, sobre diabasas (ofitas), 30SYH211272, $5 \mathrm{~m}$, 17.III.2016, Fos, Laguna \& Pérez Botella (VAL Lich. 31350, 31351; S F289257).

Flavoplaca granulosa (Müll. Arg.) Arup, Frödén \& Søchting

= Caloplaca granulosa (Müll. Arg.) J. Steiner

Saxícola calcícola recolectada en calizas compactas y dolomías, sobre superficies poco inclinadas hasta paredes verticales, en posiciones iluminadas, pero no soleadas, acompañada por especies de preferencias nitrófilas: Variospora aurantia (Pers.) Arup, Frödén \& Søchting, V. dolomiticola (Hue) Arup, Søchting \& Frödén, Verrucaria nigrescens y Xanthoria calcicola, entre otras. En las Hoces del Cabriel también convive con Caloplaca nana, citada anteriormente. Caracteriza el Caloplacetum granulosae Clauzade \& Roux 1975, de óptimo supramediterráneo, aunque alcanza el colino y el montano de la Región Eurosiberiana con una composición florística empobrecida (Roux, 1978; Renobales, 1996; Roux et al., 2009). Sin embargo, las localidades donde ha sido herborizada corresponden al piso mesomediterráneo. Diversas fuentes bibliográficas (Navarro-Rosinés, 1992; Nimis, 2016) indican su preferencia por posiciones por las que discurre la escorrentía superficial, comportamiento no observado en los ejemplares estudiados.

Especie de distribución principalmente mediterránea, que se extiende por Europa central hasta las Islas Británicas. También se conoce en Norteamérica (Esslinger, 2016). Citada de forma escasa y dispersa en España (Hladun \& Llimona, 2002-2014), las referencias conocidas la sitúan en Cantabria, País Vasco (Renobales, 1996), Madrid (Barreno \& Merino, 1981), Aragón (Etayo, 2010), Cataluña (Navarro-Rosinés, 1992), Murcia (Egea, 1984, 1985) y Andalucía (Burgaz, 2014).
España, Valencia: Venta del Moro, PN de las Hoces del Gabriel, Hoz del Rabo de la Sartén, sobre paredes calizas verticales, 30SXJ288725, 610 m, 19.V.2010, Fos (VAL Lich. 27314). Alicante: Alfafara, PN de la Serra de Mariola, entre la Font de Tarragó y la Cova de la Font, sobre roca caliza, 30SYH128939, 1000 m, 5.II.2015, Fos (VAL Lich. 307886).

Hydropunctaria maura (Wahlenb.) C. Keller, Gueidan \& Thüs

= Verrucaria maura Wahlenb. in Ach.

Especie marina, abundante en el horizonte litoral superior hasta la franja inferior del supralitoral, siempre afectada por las salpicaduras de las olas y el hálito marino, tanto sobre rocas calcáreas como silíceas (Renobales, 1996; Paz-Bermúdez et al., 1999, 2005). La acumulación de talos forma un estrato negro continuo característico de este horizonte costero que marca el paso al ambiente terrestre. Esta comunidad corresponde al Verrucarietum maurae Klem. 1955, en la que también aparece con frecuencia Hydropunctaria amphibia (= Verrucaria amphibia Clemente ex Ach.) (Renobales \& Noya, 1993). Ambas especies muestran una elevada variabilidad morfológica que dificulta su correcta diferenciación. Para separar los ejemplares recolectados en la Isla de Tabarca (Alicante), donde conviven sobre las metabasitas de la costa septentrional, se han considerado los caracteres establecidos por Renobales \& Noya (1991) para la discriminación taxonómica de ambas especies. Los ejemplares asignados a $H$. maura presentan esporas más anchas $(6-9 \mu \mathrm{m})$ y talos con borde delgado (no abrupto) y franja hipotalina de color crema más o menos desarrollada.

Los estudios moleculares de las especies marinas de Hydropunctaria C. Keller, Gueidan \& Thüs en el noroeste de Europa (Orange, 2012) han confirmado que la delimitación aceptada para $H$. maura por autores recientes incluye otras especies semicrípticas que han obligado a la tipificación de los táxones conocidos [H. maura y $H$. aracti$n a$ (Wahlenb.) Orange] y a la descripción de otros nuevos ( $H$. oceanica Orange y $H$. orae Orange). Las dificultades para su correcta identificación utilizando únicamente caracteres morfológicos, las discrepancias morfológicas apuntadas y la existencia de una Hydropunctaria sin describir en el litoral mediterráneo (S. Pérez-Ortega, com. pers.) pueden 
plantear dudas sobre la identidad del material estudiado respecto a $H$. maura s. str. No obstante, se ha decido mantener la identidad de los ejemplares en espera de un estudio pormenorizado que incluya su comparación molecular con material atlántico.

$\mathrm{Su}$ calificación como especie cosmopolita, frecuente en las costas rocosas de muchas partes del mundo, debe ser revisada con estudios equivalentes en un ámbito geográfico más extenso. En su delimitación actual, se trata de una especie ampliamente distribuida y a menudo abundante en las costas rocosas atlánticas europeas (Paz-Bermúdez et al., 2005; Orange, 2012). Su presencia en el Mediterráneo, donde resulta muy rara y confinada a sustratos silíceos (Nimis, 2016), también debe ser confirmada. Citada con frecuencia en las costas atlánticas y cantábricas españolas (Hladun \& Llimona, 20022014), no hay constancia previa de su presencia en la costa mediterránea ibérica.

España, Alicante: Alicante, Isla de Tabarca, extremo occidental de La Seca, sobre metabasitas, 30SYH211272, 1 m, 17.III.2016, Fos, Laguna \& Pérez Botella (VAL_Lich. 31308).

\section{Lecania sambucina (Körb.) Arnold}

Cortícola, parece preferir las comunidades liquénicas sobre viejos árboles caducifolios con cortezas rugosas y enriquecidas en nutrientes, como Sambucus L., Populus L., Sorbus L., Salix L., Buxus L. o Fagus L., normalmente en localidades umbrosas y húmedas (Etayo, 1989; Boqueras, 2000; Reese Næsborg, 2008). El material estudiado fue recolectado sobre un ejemplar añoso de Juniperus thurifera en un sabinar albar (Juniperetum hemispherico-thuriferae) de estructura característica, acompañada por especies de preferencias nitrófilas, como Xanthoria parietina (L.) Th. Fr., Physcia adscendens H. Olivier, Phaeophyscia orbicularis (Neck.) Moberg, Lecidella elaeochroma, Caloplaca cerina (Hedw.) Th. Fr. y C. haematites (Chaub. ex St.-Amans) Zwackh.

Diversos autores la han incluido como sinónimo de Lecania cyrtella (Ach.) Th. Fr. (Clauzade \& Roux, 1985; Smith et al., 2009) y así se recoge en el Index Fungorum; sin embargo, los resultados moleculares y morfológicos confirman su validez como especie independiente (Reese Næsborg, 2008), caracterizada por sus ascos con 12-16 esporas. El criterio taxonómico propuesto en estas obras básicas ha condicionado una escasez de datos florísticos que impide conocer su distribución general, aunque probablemente esté muy extendida por toda Europa (Nimis, 2016). En España, sólo ha sido citada expresamente en Tarragona (Boqueras, 2000) y Navarra (Etayo, 1989), aunque es muy probable que algunas de las numerosas referencias a Lecania cyrtella (Hladun \& Llimona, 2002-2014) correspondan a este taxon.

España, Valencia: Ademuz, Loma del Pino Mocho, alrededores de la Barraca del Tío Manchego, sobre Juniperus thurifera, 30TXK521340, 1100 m, 17.IX.2017, Fos (VAL_Lich. 30800).

Lecanora rupicola subsp. subplanata (Nyl.) Leuckert \& Poelt

Saxícola sobre rocas silíceas y metamórficas, coloniza superficies de poco a muy inclinadas, incluso extraplomadas, en posiciones bien iluminadas, aunque no expuestas a la radiación solar directa, y algo enriquecidas en compuestos nitrogenados (Egea \& Llimona, 1984; Hladun, 1985). El material ha sido herborizado en la Sierra del Toro (SO de Castellón), un área dominada por los sustratos de naturaleza caliza, en la que, no obstante, son frecuentes las areniscas superficialmente descarbonatadas por efecto de las lluvias. Sobre este tipo de sustrato convive con otras especies de óptimo silicícola [Xanthoparmelia gr. pulla, Rhizocarpon geographicum (L.) DC.] y con elementos calcícolas típicos [Acarospora cervina A. Massal., Circinaria calcarea (L.) A. Nordin, Savić \& Tibell, $\mathrm{Ca}$ loplaca erythrocarpa (Pers.) Zwackh, Verrucaria nigrescens, etc.].

La filogenia molecular del grupo de Lecanora rupicola (L.) Zahlbr. de Grube et al. (2004) no aclara el valor taxonómico de las cuatro subespecies de Lecanora rupicola establecidas por Leuckert \& Poelt (1989) en función de los metabolitos secundarios que presentan. Lecanora rupicola subsp. subplanata se caracteriza por la presencia en la médula de ácido tiofánico, responsable de la reacción positiva al hipoclorito $(\mathrm{C}+\mathrm{y} \mathrm{KC}+$ naranja $)$.

Distribuida de forma dispersa por el continente europeo, su área se extiende hasta Oriente próximo (Galun \& Mukhtar, 1996; John et al., 2004; Oran et al., 2018). Las escasas referencias ibéricas la sitúan en Castilla y León (López de Silanes et al., 1998), Navarra (Etayo, 1997), Aragón (Etayo, 2010), Cataluña (Hladun et al., 1986) y Murcia (Llimona \& Egea, 1984; Egea \& Llimona, 1997). 
España, Castellón: El Toro, Sierra del Toro, cerca del Corral del Gordito, sobre roca caliza, 30SXK880205, 1435 m, 25.VII.2013, Fos (VAL Lich. 30451).

Leproplaca chrysodeta (Vain.) J. R. Laundon ex Ahti

Especie subtropical templada a húmeda que coloniza principalmente superficies calcáreas fuertemente inclinadas o extraplomadas, aunque también crece sobre musgos epilíticos y en la zona baja de viejos troncos de forófitos muy diversos, incluso coníferas (Boqueras, 2000; Etayo, 1989, 1990; Nimis, 2016) $\mathrm{y}$, ocasionalmente sobre rocas silíceas (Terrón et al., 2000), penetrando incluso en entornos urbanos (Marcos Laso, 2001). Entre las muestras estudiadas, también se encuentran ejemplares terrícolas. Estos datos sugieren una elevada amplitud ecológica que le permite colonizar sustratos muy diversos en un amplio rango altitudinal.

Ampliamente distribuida en Europa por debajo del piso subalpino (Laundon, 1974; Nimis, 2016), aunque poco citada en España (Hladun \& Llimona, 2002-2014), donde debe ser más común de lo que indican las referencias. Se diferencia de Leproplaca xantholyta (Nyl.) Nyl. en su falta de lobulación, carácter que en ocasiones resulta difícil de diferenciar, y, sobre todo, por su color ocre, no amarillo intenso.

España, Castellón: Montanejos, Valle del río Mijares, acceso a la Cueva Negra, sobre roca caliza, 30TYK101387, 550 m, 14.X.2017, Fos \& F.S. Fos (VAL Lich. 31596); Orpesa, Cala dels Retors, sobre roca caliza, 31TBE556404, $10 \mathrm{~m}, 7$. V.2014, Fos (VAL Lich. 30774); La Mata de Morella, valle del río Cantavieja, en acumulaciones de suelo entre rocas, en muro de piedra seca, 30TYK301993, 775 m, 6.V.2015, Fos (VAL Lich. 31636).

\section{Leproplaca cirrochroa (Ach.) Arup}

\section{= Caloplaca cirrochroa (Ach.) Th. Fr.}

Saxícola sobre calizas duras y dolomías, coloniza paredes fuertemente inclinadas o extraplomadas, en posiciones sombreadas, protegidas de la lluvia y eutrofizadas, en un amplio rango altitudinal que abarca todos los pisos de vegetación. Ocasionalmente fértil (Navarro-Rosinés \& Roux, 1994), todos los ejemplares observados carecen de apotecios.

De distribución probablemente holártica, se encuentra ampliamente distribuida en Europa, resultando frecuente en la Región Mediterránea (Gaya, 2009;
Nimis, 2016) hasta el norte de África (Seaward \& Sipman, 2006). También está presente en Norteamérica (Esslinger, 2016), en Siberia (Davydov, 2014) y en el Himalaya (Poelt \& Hinteregger, 1993). Citada con frecuencia en la Península Ibérica (Hladun \& Llimona, 2002-2014).

España, Castellón: Morella, Muntanyes de Vallivana, Barranc del Tell, sobre roca caliza, 31TBE472949, 950 m, 8.IX.2016, Fos, Gómez-Serrano, Agueras \& Sanchis (VAL_Lich. 31143).

Myriolecis reuteri (Schaer.) Śliwa, Zhao Xin \& Lumbsch

= Lecanora reuteri Schaer.

Saxícola calcícola de zonas elevadas, forma parte de comunidades poco diversas que cubren amplias superficies en la porción inferior de las paredes escarpadas o verticales protegidas de la insolación y con cierto grado de humedad de la Sierra de Aitana (Alicante), por encima de los 1100 metros de altitud.

Distribuida de forma dispersa por las montañas elevadas del el centro y sur de Europa, desde el sur de Rusia hasta la Península Ibérica (Clauzade \& Roux, 1975; Nimis, 2016; Faltynowicz \& Kossowska, 2016; Roux et al., 2017; GBIF, 2018). Muy poco citada en España, sólo se conoce en Cantabria (Pérez-Ortega \& Álvarez-Lafuente, 2006a), interior de Murcia (Egea, 1985) y norte de Navarra (Etayo \& Calvo, MA-Lichen 3841; GBIF, 2018). Roux et al. (2017) indican su presencia en la vertiente francesa de los Pirineos.

España, Alicante: Confrides, Serra d'Aitana, Les Moles, en paredes verticales de roca caliza, 30SYH383835, 1130 m, 11.XII.2015, Fos \& Gómez-Serrano (VAL_Lich. 31276, 31277).

Ochrolechia alboflavescens (Wulfen) Zahlbr.

Cortícola, preferentemente sobre de coníferas, más raramente sobre leño y caducifolios de cortezas ácidas. Parece tener su óptimo en los bosques montanos y subalpinos de la Región Eurosiberiana, aunque aparece ocasionalmente en el piso colino. En la Región Mediterránea queda restringida al piso oromediterráneo (Etayo, 1989; Brodo, 1991; Tönsberg, 1992; Boqueras et al., 1999; Nimis, 2016). Probablemente, las referencias de Sarrión et al. (1993) y Fos (1998), que la encuentran respectivamente en melojares y encinares y en alcornocales mesomediterráneos de notable influencia atlántica, no correspondan a esta especie, porque las 
condiciones ecológicas son prácticamente opuestas a las descritas. El material estudiado fue recolectado sobre pino albar (Pinus sylvestris) en un pinar oromediterráneo (Sabino-Pinetum sylvestris) bastante abierto, donde alcanzaba coberturas elevadas en posiciones expuestas y bien iluminadas.

Esta especie boreal-montana, ampliamente distribuida en Europa, queda restringida a la alta montaña en Europa central y en los territorios mediterráneos. En España se extiende principalmente por los Pirineos y la cordillera Cantábrica (Boqueras et al., 1999), con algunas localidades dispersas en el Sistema Ibérico (Fos, 2002; Etayo, 2010), el Sistema Central (Ibáñez \& Burgaz, 1995; Boqueras et al., 1999) y las Sierras Béticas (Aragón et al., 2006; Burgaz et al., 2017).

España, Valencia: Puebla de San Miguel. Sierra de Javalambre, MRF "Pino de Vicente Tortajada", sobre Pinus sylvestris, 30TXK616376, $1700 \mathrm{~m}$, 29.VII.2006, Fos (VAL_Lich. 27360).

\section{Rhizocarpon umbilicatum (Ramond) Flagey}

Saxícola, coloniza rocas calcáreas y más raramente silíceas ricas en bases o ligeramente carbonatadas. Muestra preferencia por paredes verticales o fuertemente inclinadas, poco soleadas y húmedas, normalmente de orientación norte. También se instala en superficies más o menos horizontales, expuestas y eutrofizadas (Renobales, 1996; Etayo, 2010). El material estudiado aparece precisamente en estas condiciones ecológicas, acompañado por Acarospora cervina, Circinaria calcarea, C. contorta subsp. hoffmanniana (S. Ekman \& Fröberg ex R. Sant.) Zhdanov, Variospora dolimiticola (Hue) Arup, Søchting \& Frödén y otras especies de preferencias nitrófilas, en una localidad supramediterránea continental, por encima de los $1500 \mathrm{~m}$ de altitud.

Especie principalmente circumpolar ártico-alpina (Nimis, 2016) ampliamente distribuida por las áreas calcáreas europeas. Poco citada en la Península Ibérica (Hladun \& Llimona, 2002-2014), debe ser más abundante de lo que indican los registros actuales.

España, Valencia: Puebla de San Miguel, PN de Puebla de San Miguel, Sierra de Javalambre, MRF "Barranco del Saladillo", sobre roca caliza, 30TXK639373, 1687 m, 25.V.2011, Fos, Navarro \& Oltra (VAL_Lich. 28136).
Staurothele hymenogonia (Nyl.) Th. Fr.

Saxícola calcícola de talo endolítico, crece sobre calizas, dolomías y areniscas calcáreas, normalmente en posiciones soleadas, más o menos enriquecidas en compuestos nitrogenados y cercanas al suelo. También forma parte de la composición florística que coloniza monumentos y elementos arqueológicos (Ariño \& Saiz-Jiménez, 1997). Aparece en un amplio rango altitudinal que se extiende desde el litoral hasta el supramediterráneo, por encima de los $1000 \mathrm{~m}$ de altitud. El material ha sido recolectado en guijarros dispersos sobre el suelo, un tipo de sustrato en el que resulta frecuente (Casares \& Llimona, 1986; Navarro-Rosinés, 1992; Smith et al., 2009; Shivarov \& Stoykov, 2012).

Distribuida por toda Europa, desde la Región Boreal hasta la Mediterránea, su área se extiende hasta Asia (Seaward et al., 2004; Yazıc1 et al., 2010) y Norteamérica (Esslinger, 2016). Poco citada en España, probablemente sea mucho más abundante de lo que indican las referencias conocidas y haya pasado desapercibida debido a su pequeño tamaño y a sus preferencias litófilas, que la hacen especialmente frecuente en las piedras esparcidas por el suelo. Se conoce en Castilla y León (Pérez-Ortega \& Álvarez-Lafuente, 2006b), Castilla-La Mancha (Carballal \& Giménez-Coral, 1981), Cataluña (Navarro-Rosinés, 1992) y Andalucía (Burgaz, 2014).

España, Alicante: Alcoy, Les Solanetes, El Fardatxo, en guijarros calizos, 30SYH150848, $750 \mathrm{~m}$, 19.V.2009, Fos (VAL_Lich. 26682).

\section{Verrucaria halizoa Leight.}

Saxícola marina, resulta frecuente en el horizonte litoral superior de las costas atlánticas, especialmente en los niveles medios e inferiores, sobre todo tipo de litologías. Coloniza paredes verticales y subverticales, tanto en posiciones expuestas e iluminadas como en protegidas y sombreadas, acompañada entre otras por Wahlenbergiella striatula (Wahlenb.) Gueidan \& Thüs, W. mucosa (Wahlenb.) Gueidan \& Thüs, Hydropunctaria amphibia e H. maura (Renobales, 1996; Paz-Bermúdez et al., 1999; 2000; Orange, 2013). En la Isla de Tabarca (Alicante), aparece de forma muy puntual en los acantilados litorales de orientación norte sobre rocas básicas de origen volcánico (metabasitas), acompañada por $H$. amphibia. 
Ampliamente extendida por toda la costa atlántica europea (Smith et al., 2009); también se conoce en Norteamérica y Asia (Orange, 2013; Esslinger, 2016). Citada con frecuencia en todo el litoral cantábrico (Renobales \& Noya, 1993; Renobales, 1996; Paz-Bermúdez et al., 1999, 2000); sin embargo, en el Mediterráneo sólo ha sido citada previamente por Navarro-Rosinés (1992, sub Verrucaria microspora auct. non Nyl.) en Tarragona.

España, Alicante: Alicante, Isla de Tabarca, extremo occidental de La Seca, sobre metabasitas 30SYH211272, 1 m, 17.III.2016, Fos, Laguna \& Pérez-Botella (VAL_Lich. 31310, 31311).

\section{Xanthoria aureola (Ach.) Erichsen}

Los caracteres morfológicos y moleculares han confirmado la independencia taxonómica de Xanthoria aureola, tanto de $X$. parietina, con la que muestra importantes similitudes morfológicas, especialmente cuando conviven en zonas litorales, como de $X$. calcicola, de la que era considerada un mero sinónimo (Lindblom \& Ekman, 2005, 2012). Diversos autores (Lindblom et al., 2005; Smith et al., 2009; Fiorentino, 2012; Nimis, 2016; Roux et al., 2017) sitúan su óptimo en hábitats marítimos, sobre rocas silíceas básicas, en posiciones expuestas y enriquecidas en nutrientes; no obstante, Smith et al. (2009) también indican su presencia sobre calizas en áreas elevadas de interior y ha sido recientemente recolectada en la Serranía de Ronda (Málaga) sobre roca silíceas (gneiss) a $1255 \mathrm{~m}$ de altitud (Burgaz et al., 2017). El material estudiado aparece en condiciones ecológicas que se corresponden con estas últimas: roquedos calcáreos en áreas interiores de Valencia, cercanas a los 1000 metros de altitud, en posiciones expuestas, bien iluminadas y algo nitrificadas. Los caracteres morfológicos (lóbulos crenulados, estrechos, superpuestos en el centro del talo, ausencia de apotecios y de isidios y superficie rugosa por la presencia de cristales) se corresponden con los indicados en la bibliografía para diferenciarla de $X$. parietina y $X$. calcicola.

Especie mediterránea-atlántica (Nimis, 2016) citada en Europa occidental, desde la Región Mediterránea hasta las Islas Británicas y la Península Escandinava, aunque su distribución permanece mal conocida debido a su tratamiento taxonómico como sinónimo de $X$. calcicola durante muchos años. La Check-List de los líquenes ibéricos (Hladun \& Llimona, 2002-2014) mantiene este criterio, aunque diferencia numerosas citas atribuidas originalmente a $X$. aureola. Resulta complicado poder discriminar qué referencias corresponden realmente a este taxon en su delimitación actual y por esta razón, se omite cualquier comentario sobre su distribución en España.

España, Valencia: Chera, PN de Chera-Sot de Chera, Sierra del Negrete, MRF "Pico de Ropé", sobre roca caliza, 30SXJ733878, 980 m, 9.IX.2015, Fos, Oltra \& Gómez-Serrano (VAL_Lich. 31270).

\section{DISCUSIÓN}

Los estudios florísticos en cualquier ámbito geográfico son fundamentales para identificar su riqueza y diversidad específica. Son la fuente de información básica para determinar la abundancia de las especies, sus preferencias ecológicas o sus patrones de distribución. Sin embargo, de un tiempo a esta parte, este tipo de estudios vienen experimentado una progresiva devaluación que está provocando una alarmante reducción. Para confirmar esta afirmación, sólo hay que revisar el número de publicaciones de flora en los listados anuales de la bibliografía botánica ibérica (líquenes): cinco artículos en 2014 (Burgaz, 2015b), seis en 2015 (Burgaz, 2016) y cuatro en 2016 (Burgaz, 2017). La mayoría de los trabajos incluyen novedades florísticas a escala local, regional e, incluso, nacional, pero desgraciadamente el volumen de información es demasiado escaso para corregir la principal consecuencia de esta situación: la enorme extensión de los territorios sin información alguna sobre su diversidad liquénica (Fos et al., 2014; Fos, 2016). En este sentido, no puede quedar sin mención el proyecto de elaboración de la Lista Roja de los hongos liquenizados y liquenícolas de España y Portugal (Atienza et al., 2017). La correcta evaluación del estado de conservación de las especies amenazadas exige la información que generan los estudios florísticos, máxime cuando el número de localidades y/o poblaciones constituye el criterio de la UICN más utilizado para asignar categorías de amenaza a los líquenes (Atienza \& Segarra, 2000; Martínez et al., 2003; Liška et al., 2008; Nascimbene et al., 2013).

Este trabajo se enmarca en esta necesaria línea de trabajo con una veintena de especies que son citadas por primera vez en la Comunitat Valenciana. 
Estas nuevas poblaciones no sólo incrementan la riqueza florística regional, también contribuyen a mejorar el conocimiento corológico de diversas especies raras o poco citadas en España (Calicium notarisii, Caloplaca nana, Dirina fallax, Lecania sambucina, Lecanora rupicola subsp. subplanata, Myriolecis reuteri, Rhizocarpon umbilicatum, Staurothele hymenogonia, Xanthoria aureola) o en los territorios mediterráneos ibéricos (Bryoria capillaris, Hydropunctaria maura, Ochrolechia alboflavescens, Verrucaria halizoa). Se aportan incluso dos nuevos taxones a la flora liquénica española: Candelariella commutata, recientemente descrito y probablemente mucho más abundante de lo que indican las referencias actuales, y Catinaria neuschildii, una especie extraordinariamente rara en toda su área de distribución.

\section{AGRADECIMIENTOS}

Quiero expresar mi agradecimiento a todos los compañeros, colegas y amigos que me han acompañado en las numerosas campañas de prospección y que me han facilitado información adicional y material gráfico. Su motivadora colaboración ha permitido localizar muchas de las especies tratadas y, sobre todo, alcanzar el nivel de conocimiento actual de la flora liquénica valenciana. También agradecer al Dr. A. Tehler (Swedish Museum of Natural History) su revisión del material de Dirina fallax, a V. Atienza (Universitat de València) por la gestión y conservación de las muestras en el VAL_Lich. y a N. Fabuel (Ceteck Tecnológica) por toda la información relativa a los líquenes en el BDBCV. Finalmente, agradecer al Dr. S. PérezOrtega, al Dr. E. Llop y al Dr. L. Sáez su minuciosa revisión y sus valiosos comentarios y sugerencias que han contribuido a mejorar el manuscrito original.

\section{REFERENCIAS BIBLIOGRÁFICAS}

Alonso, F. \& Egea, J. M. 1999. Líquenes epífitos de algunas localidades costeras del sur y sureste de España. Anales de Biología 22: 125-134.

Aragón, G. 2002. Líquenes del Parque Natural de Cazorla, Segura y Las Villas (Jaén, España). Tesis Doctoral, Universidad Complutense de Madrid, Madrid.

Aragón, G., Martínez, I. \& Belinchón, R. 2006. Aportación al conocimiento de los líquenes epífitos de Pinus halepensis, $P$. nigra, $P$. pinaster y $P$. sylvestris en la Península Ibérica. Botanica Complutensis 30: 61-70.

Aragón, G., Martínez, I. \& Burgaz, A. R. 1999. Líquenes epifíticos de la Sierra del Tremedal (Guadalajara-Teruel). Teruel 87: 95-110.

Aragón, G., Martínez, I. \& Burgaz, A. R. 2001. Macrolíquenes de Castilla-La Mancha. Instituto de Estudios Manchegos, Ciudad Real.
Ariño, X. \& Sáiz-Jiménez, C. 1997. Deterioration of the Elephant Tomb (Necropolis of Carmona, Seville, Spain). International Biodeterioration \& Biodegradation 40: 233239. https://doi.org/10.1016/S0964-8305(97)00034-6

Atienza, V. 2002. Two new species of Minutoexcipula from Spain. Bibliotheca Lichenologica 82: 141-152.

Atienza, V., Araujo, E., Burgaz, A. R. et al. 2017. Towards a Red List of lichen-forming and lichenicolous fungi in Spain and Portugal. British Lichen Society Bulletin 120: 43-53.

Atienza, V., Calatayud, V. \& Hawksworth, D. 2003. Notes on the genus Polycoccum (Ascomycota, Dacampiaceae) in Spain, with a key to the species. Lichenologist 35: 125-135. https://doi.org/10.1016/S0024-2829(03)00014-8

Atienza, V. \& Fos, S. 2011. Hongos liquenizados y liquenícolas del Parque Natural de la Albufera. Butlleti de la Societat Micologica Valenciana 16: 3-39.

Atienza, V., Pérez-Ortega, S. \& Etayo, J. 2009. Two new conidial lichenicolous fungi from Spain indicate the distinction of Lichenodiplis and Minutoexcipula. Lichenologist 41: 223-230. https://doi.org/10.1017/S0024282909008378

Atienza, V. \& Segarra, J. G. 1999a. A first approximation checklist of the lichens of the Valencian Community. Flora Mediterranea 9: 231-268.

Atienza, V. \& Segarra, J. G. 1999b. Fragmenta Chorologica Occidentalia, Lichenes, 7040-7082. Anales del Jardín Botánico de Madrid 57: 148-151.

Atienza, V. \& Segarra, J. G. 2000. Preliminary Red List of the lichens of the Valencian Community (Eastern Spain). Forest Snow and Landscape Research 75: 391-400.

Atienza, V., Segarra, G. \& Laguna, E. 2001. Propuesta de microrreservas vegetales. Una alternativa para la conservación de líquenes en la Comunidad Valenciana. Botanica Complutensis 25: 115-128.

Barreno, E. \& Merino, A. 1981. Catálogo liquénico de las calizas de Madrid (España). Lazaroa 3: 247-268.

BDBCV [Banco de Datos de Biodiversidad de la Comunitat Valenciana] 2017. Consultado el 29 de diciembre de 2017, en http://bdb.cma.gva.es

Boom, P. P. G. van den \& Etayo, J. 2014. New records of lichenicolous fungi and lichenicolous lichens from the Iberian Peninsula, with the description of four new species and one new genus. Opuscula Philolichenum 13: 44-79.

Boom, P. P. G. van den \& Palice, Z. 2006. Some interesting lichens and lichenicolous fungi from the Czech Republic. Czech Mycology 58: 105-116.

Boqueras, M. 2000. Líquens epifits i fongs liquenicoles del sud de Catalunya. Flora i comunitats. Institut d'Estudis Catalans, Barcelona.

Boqueras, M., Barbero, M. \& Llimona, X. 1999. El género Ochrolechia A. Massal. (Pertusariaceae, líquenes) en España y Portugal. Cryptogamie Mycologie 20: 303-328. https://doi.org/10.1016/S0181-1584(00)88858-2

Boqueras, M. \& Llimona, X. 2003. The genus Pertusaria (lichenised Ascomycotina) on the Iberian Peninsula and Balearic Islands. I. Subgenus Pertusaria. Mycotaxon 88: 471-492.

Brodo, I. M. 1991. Studies in the lichen genus Ochrolechia. 2. Corticolous species of North America. Canadian Journal of Botany 69: 733-772. https://doi.org/10.1139/b91-099

Burgaz, A. R. 2011. Asientos de flora liquenológica ibérica: Massalongiaceae y Placynthiaceae. Clementeana 13: 15-49.

Burgaz, A. R. 2014. Líquenes de Andalucía (S de España): catálogo bibliográfico y nuevos datos del NW del área. Botanica Complutensis 38: 53-88. http://dx.doi.org/10.5209/ rev_BOCM.2014.v38.45775 
Burgaz, A. R. 2015a. Asientos de flora liquenológica ibérica: Cladoniaceae. Clementeana 16: 3-158.

Burgaz, A. R. 2015b. Bibliografía botánica ibérica, 2014. Líquenes. Botanica Complutensis 39: 129-131.

Burgaz, A. R. 2016. Bibliografía botánica ibérica, 2015. Líquenes. Botanica Complutensis 40: 203-207.

Burgaz, A. R. 2017. Bibliografía botánica ibérica, 2016. Líquenes. Botanica Complutensis 41: 109-113. http://dx.doi. org/10.5209/BOCM.56869

Burgaz, A. R., Atienza, V., Chesa, M. J. et al. 2017. Lichens and lichenicolous fungi of Serranía de Ronda (MálagaCádiz), southern Spain. Botanica Complutensis 41: 9-28. https://doi.org/10.5209/BOCM.56861

Calatayud, V. 1998. Liquenes y hongos liquenícolas de rocas no carbonatadas en el Sistema Ibérico Oriental e Islas Columbretes. Tesis Doctoral, Universitat de València, València.

Calatayud, V. \& Barreno, E. 2003. A new Lichenostigma on vagrant Aspicilia species. Lichenologist 35: 279-285. https://doi.org/10.1016/S0024-2829(03)00052-5

Calatayud, V. \& Etayo, J. 2001. Five new species of lichenicolous conidial fungi from Spain. Canadian Journal of Botany 79: 223-230. https://doi.org/10.1139/b00-144

Calatayud, V. \& Navarro-Rosinés, P. 2001. Bellemerella acarosporae and $B$. polysporinae (Verrucariales), two new species of lichenicolous fungi from Spain. Nova Hedwigia 72: 473-478.

Calatayud, V., Navarro-Rosinés, P. \& Hafellner, J. 2002. A synopsis of Lichenostigma subgen. Lichenogramma (Arthoniales), with a key to the species. Mycological Research 106: 1230-1242. https://doi.org/10.1017/S095375620200655X

Calatayud, V., Navarro-Rosinés, P. \& Hafellner, J. 2013. Contributions to a revision of Cercidospora (Dothideales), 2: Species on Lecanora s. l., Rhizoplaca and Squamarina. Mycosphere 4: 539-557. https://doi.org/10.5943/mycosphere $/ 4 / 3 / 8$

Calatayud, V. \& Sanz, M. J. 2000. Guía de líquenes epifitos en las parcelas del sistema Pan-Europeo para el seguimiento intensivo y continuo de los sistemas forestales (Red CE de Nivel II) en España. Parques Nacionales (Ministerio de Medio Ambiente), Madrid.

Calatayud, V. \& Triebel, D. 2001. Stigmidium acetabuli (Dothideales sens. lat.), a new lichenicolous fungus on Pleurosticta acetabulum. Bibliotheca Lichenologica 78: 27-33.

Calatayud, V. \& Triebel, D. 2003. Three new species of Stigmidium (lichenicolous ascomycetes) on Acarospora and Squamarina. Lichenologist 35: 103-116. https://doi. org/10.1016/S0024-2829(02)00097-X

Carballal, R. 2013. El género Roccella en la Península Ibérica y las Islas Baleares. Botanica Complutensis 37: 13-20. https://doi.org/10.5209/rev BOCM.2013.v37.42263

Carballal, R. \& Giménez-Coral, R. M. 1981. Líquenes de las «calizas de los páramos» de la Alcarria Occidental (Guadalajara). Trabajos del Departamento de Botánica y Fisiología Vegetal. Facultad de Ciencias Biológicas. Universidad [Complutense] de Madrid 11: 59-71.

Carvalho, P. 2009. Estudo corológico do genero Collema na Peninsula Ibérica. Portugaliae Acta Biologica 23: 37-146.

Carvalho, P. 2012. Flora Liquenológica Ibérica 10 - Collema. Sociedad Española de Liquenología, Pontevedra.

Casares, M. \& Llimona, X. 1986. La clase Verrucarietea nigrescentis Wirth 1980 en las calizas béticas de la provincia de Granada. Cryptogamie, Bryologie et Lichénologie 7: 103-127.
Clauzade, G. \& Roux, C. 1975. Etude écologique et phytosociologique de la végétation lichénique des roches calcaires non altérées dans les régions méditerranéenne du sud-est de la France. Bulletin du Musée d'Histoire Naturelle de Marseille 35: 153-208

Clauzade, G. \& Roux, C. 1985. Likenoj de Okcidenta Europo. Ilustrita determinlibro. Bulletin de la Société Botanique du Centre-Ouest (nouv. sér.) num. spéc. 7: 1-893.

Coppins, A. M. \& Coppins, B. J. 2002. Indices of ecological continuity for woodland epiphytic lichen habitats in the British Isles. British Lichen Society, London.

Crespo, A., Blanco, O., Llimona, X., Ferencová, Z. \& Hawksworth, D. 2004. Coscinocladium, an overlooked endemic and monotypic Mediterranean lichen genus of Physciaceae, reinstated by molecular phylogenetic analysis. Taxon 53: 405-414. https://doi.org/10.2307/4135618

Davydov, E. A. 2014. The first checklist of lichens, lichenicolous and allied fungi of Altaisky krai (Siberia, Russia). Mycotaxon 127: 231. http://dx.doi.org/10.5248/127.231

Divakar, P. K., Molina, M. C., Lumbsch, H. T. \& Crespo, A. 2005. Parmelia barrenoae, a new lichen species related to Parmelia sulcata (Parmeliaceae) based on molecular and morphological data. Lichenologist 37: 37-46. https://doi. org/10.1017/S0024282904014641

Egea, J. M. 1984. Contribución al conocimiento del género Caloplaca Th. Fr. en España: Especies saxícolas. Collectanea Botanica 15: 173-204.

Egea, J. M. 1985. Líquenes calcícolas y terrícolas de las Sierras de Pedro Ponce y Quípar (NW de Murcia, España). Anales de Biología 6 (Biología Vegetal 1): 19-27.

Egea, J. M. 1989. Las comunidades liquénicas saxícolas, ombrófobas, litorales, del Suroeste de Europa y Norte de África. Studia Geobotanica 9: 73-152.

Egea, J. M. \& Llimona, X. 1984. Las comunidades liquénicas saxícolas ombrófobas de la costa del SE de España comprendidas entre el Penyal d'Ifac (Alicante) y Almería. Collectanea Botanica 15: 205-219.

Egea, J. M. \& Llimona, X. 1994. La flore et la végétation liquéniques des laves acides du parc natural de la Sierra del Cabo de Gata (SE de l'Espagne) et des régions voisines. Bulletin de la Société Linnéenne de Provence 45: 263-281.

Egea, J. M. \& Llimona, X. 1997. Sobre la flora y vegetación liquénicas de las lavas básicas del sureste de España. Acta Botanica Malacitana 22: 5-11.

Egea, J. M. \& Rowe, J. G. 1987. Lichenological excursion in North Africa. I. Silicicolous lichens in Morocco. Collectanea Botanica 17: 27-45. https://doi.org/10.3989/collectbot.1988.v17.153

Esslinger, T. 2016. A cumulative checklist for the lichenforming, lichenicolous and allied fungi of the Continental United States and Canada, Version 21. Opuscula Philolichenum 15: 136-390.

Etayo, J. 1989. Liquenes epifitos del Norte de Navarra. Tesis Doctoral, Universidad de Navarra, Pamplona.

Etayo, J. 1990. Ensayo de la vegetación liquénica epífita del Norte de Navarra. Principe de Viana (Suplemento de Ciencias) 10: 39-71.

Etayo, J. 1997. Líquenes de roquedos silíceos en los Pirineos occidentales. Naturzale 12: 123-148.

Etayo, J. 2010. Líquenes y hongos liquenícolas de Aragón. Guineana 16: 1-501.

Etayo, J. \& Breuss, O. 1996. Líquenes y hongos liquenícolas de los Pirineos Occidentales y Norte de la Península Ibérica, IV. Cryptogamie, Bryologie et Lichénologie 17: 213-230. 
Faltynowicz, W. \& Kossowska, M. 2016. The lichens of Poland. A fourth checklist (Acta Botanica Silesiaca Monographiae, 8). Biologica Silesiae, Wrocław.

Fiorentino, J. 2011-2012. The genus Xanthoria (Teloschistaceae, Lichenised Ascomycota) in the Maltese Islands. The Central Mediterranean Naturalist 5: 9-17.

Fos, S. 1998. Líquenes epífitos de los alcornocales ibéricos. Correlaciones bioclimáticas, anatómicas y densimétricas con el corcho de reproducción. Guineana 4: 1-507.

Fos, S. 2001. Flora liquénica de la Microrreserva de las Hoyuelas y alrededores (Sinarcas, Plana de Utiel, E de Valencia). Conselleria de Medi Ambient (Generalitat Valenciana), València.

Fos, S. 2002. Estudio de la diversidad liquénica de los sabinares y pinares de la Sierra de Javalambre (Teruel). Teruel 88-89: 211-245.

Fos, S. 2016. La conservació dels fongs liquenitzats: diagnòstic d'actualitat i desafiament per al futur. In: Llibre de Resums de les IV Jornades de Conservació de Flora (Olot, 28, 29 i 30 de novembre, 2016). Fundació d'Estudis Superiors d'Olot, Olot: 11.

Fos, S., Atienza, V. \& Fabuel, N. 2014. El banco de datos de biodiversidad de la Comunitat Valenciana: portal de información sobre biodiversidad liquénica. Clementeana 15: 24-27.

Fos, S. \& Barreno, E. 2002. Fragmenta Chorologica Occidentalia, Lichenes, 8106-8141. Anales del Jardín Botánico de Madrid 59: 310-311.

Fos, S., Calatayud, A. \& Barreno, E. 2001. Diversidad liquénica asociada a fenómenos post-incendio en los alcornocales valenciano-castellonenses. Botanica Complutensis 25: $103-113$.

Fos, S. \& Giralt, M. 2009. Rinodina evae (Physciaceae, Ascomycota), a new corticolous blastidiate species from Eastern Spain. Nova Hedwigia 88: 33-40. https://doi. org/10.1127/0029-5035/2009/0088-0033

Fos, S., Gómez-Serrano, M. A., Sanchís, M. J. \& Agueras, M. 2017. Redescubrimiento del liquen Lobaria pulmonaria aparentemente extinto en la Comunidad Valenciana. Flora Montiberica 67: 114-119.

Galun, M. \& Mukhtar, A. 1996. Checklist of the lichens of Israel. Bocconea 6: 149-171.

Garrido-Benavent, I., Llop, E. \& Gómez-Bolea, A. 2013. Catálogo de los líquenes epífitos de Quercus ilex subsp. rotundifolia de la Vall d'Albaida (Valencia, España). Botanica Complutensis 37: 27-33. http://dx.doi.org/10.5209/ rev BOCM.2013.v37.42265

Garrido-Benavent, I. \& Pérez-Ortega, S. 2015. Unravelling the diversity of European Caliciopsis (Coryneliaceae, Ascomycota): Caliciopsis valentina sp. nov. and C. beckhausii comb. nov., with a worldwide key to Caliciopsis. Mycological Progress 14: 1-11. https://doi.org/10.1007/s11557015-1034-2

Gaya, E. 2009. Taxonomical revision of the Caloplaca saxicola group (Teloschistaceae, lichen-forming Ascomycota). Bibliotheca Lichenologica 101: 1-191.

Gaya, E. \& Pérez, A. 2002. Primera citació de Teloschistes lacunosus (Rupr.) Savicz al País Valencià. Butlletí de la Institució Catalana d'Història Natural 70: 48-51.

GBIF [Global Biodiversity Information Facility] 2018. GBIF. org. Consultado el 30 de enero de 2018, en GBIF Occurrence Download, https://doi.org/10.15468/dl.cvkq72.

Giralt, M. \& Nordin, A. 2002. Buellia triseptata in the Iberian Peninsula. Lichenologist 34: 89-94. https://doi. org/10.1006/lich.2002.0375
Giralt, M., Paz-Bermúdez, G. \& Elix, J. A. 2009. The saxicolous, xanthone-containing species of the genus Buellia s. l. (Physciaceae, Ascomycota) in the Iberian Peninsula. Nova Hedwigia 89: 321-334. https://doi.org/10.1127/00295035/2009/0089-0321

Grube, M., Baloch, E. \& Arup, U. 2004. A phylogenetic study of the Lecanora rupicola group (Lecanoraceae, Ascomycota). Mycological Research 108: 506-514. https:// doi.org/10.1017/S0953756204009888

Hafellner, J. 1995. A new check-list of lichens and lichenicolous fungi of insular Laurimacaronesia including a lichenological bibliography for the area. Fritschiana 5: 1-132.

Hawksworth, D. L. 2004. A fourth site for Cyphelium notarisii (Physciaceae) in Spain. Lazaroa, 25: 89-91.

Heiömarsson, S. 2001. The genus Dermatocarpon (Verrucariales, lichenized Ascomycotina) in the Nordic countries. Nordic Journal of Botany 20: 605-639. http://doi.wiley. com/10.1111/j.1756-1051.2000.tb01612.x

Hladun, N. L. 1985. Aportació a la flora, morfologia $i$ vegetació dels líquens de la part alta del Montseny. Institut d'Estudis Catalans, Barcelona.

Hladun, N. L., Gómez-Bolea, A. \& Llimona, X. 1986. Els líquens del Montseny. In: El patrimoni natural del Montseny. Servei de Parcs Naturals (Diputació de Barcelona), Barcelona: $1-10$.

Hladun, N. \& Llimona, X. 2002-2014. Checklist of the Lichens and lichenicolous fungi of the Iberian Peninsula and Balearic Islands. Universitat de Barcelona, Barcelona. Consultado el 31 de enero de 2018, en http://botanica.bio.ub.es/ checklist/checklist.htm

Ibáñez, I. \& Burgaz, A. R. 1995. Líquenes epífitos de Barco de Ávila (Ávila, España). Botanica Complutensis 20: 9-18.

John, V., Seaward, M. R. D., Sipman, H. J. M. \& Zedda, L. 2004. Lichens and lichenicolous fungi of Syria, including a first checklist. Herzogia 17: 157-177.

Laguna, E., Fos, S., Jiménez, J. \& Volis, S. 2016. Role of micro-reserves in conservation of endemic, rare and endangered plants of the Valencian region (Eastern Spain). Israel Journal of Plant Sciences 63: 320-332. https://doi.org/10.1 080/07929978.2016.1256131

Laundon, J. R. 1974. Leproplaca in the British Isles. Lichenologist 6: 102-105. https://doi.org/10.1017/S0024282974000077

Leuckert, C. \& Poelt, J. 1989. Studien über die Lecanora rupicola-Gruppe in Europa (Lecanoraceae). Nova Hedwigia 49: 121-167.

Lindblom, L. \& Ekman, S. 2005. Molecular evidence supports the distinction between Xanthoria parietina and $X$. aureola (Teloschistaceae, lichenized Ascomycota). Mycological Research 109: 187-199. https://doi.org/10.1017/ S0953756204001790

Lindblom, L. \& Ekman, S. 2012. RAPDs distinguish the lichens Xanthoria aureola and X. parietina in a mixed seashore rock population. Nova Hedwigia 94: 279-285. https:// doi.org/10.1127/0029-5035/2012/0009

Lindblom, L., Ladstein, M. H., Blom, H. H., Ekman, S. \& Timdal, E. 2005. Xanthoria aureola in Norway and a key to the species of Xanthoria s. str. in Scandinavia. Graphis Scripta 17: $12-16$

Liška, J., Palice, Z. \& Slavíková, Š. 2008. Checklist and Red List of lichens of the Czech Republic. Preslia 80: 151-182.

Llimona, X. \& Egea, J. M. 1984. La vegetación liquénica saxícola de los volcanes del Mar Menor (Murcia, SE de España). Butlletí de la Institució Catalana d'Història Natural 51: 77-99. 
Llop, E. 2003. La familia Bacidiaceae (Lecanorales) a la Península Ibèrica i les Illes Balears. Els gèneres Bacidia $i \mathrm{Ba}-$ cidina. Tesis Doctoral, Universitat de Barcelona, Barcelona.

Llop, E. 2010. Bacidia punica (Ramalinaceae), a new corticolous species from the Mediterranean region. Bryologist 113: 365-370. https://doi.org/10.1639/0007-2745-113.2.365

López de Silanes, M. E., Terrón, A. \& Etayo, J. 1998. Líquenes y hongos liquenícolas de Fuentes Carrionas, Sierra de Riaño y Valle de Liébana (N de España). Nova Acta Científica Compostelana-Bioloxía 8: 47-89.

Lumbsch, H. T. 1989. Die holarktische Vertreter der Flechtengattung Diploschistes (Thelotremataceae). The Journal of the Hattori Botanical Laboratory 66: 133-196.

Lumbsch, H. T., Ahti, T., Altermann, S. et al. 2011. One hundred new species of lichenized fungi: a signature of undiscovered global diversity. Phytotaxa 18: 1-127. http:// dx.doi.org/10.11646/phytotaxa.18.1.1

Marcos Laso, B. 2001. Biodiversidad y colonización liquénica de algunos monumentos en la ciudad de Salamanca (España). Botanica Complutensis 25: 93-102.

Martínez, I., Aragón, G., Sarrión, F. J., Escudero, A., Burgaz, A. R. \& Coppins, B. J. 2003. Threatened lichens in central Spain (saxicolous species excluded). Cryptogamie Mycologie 24: 73-97.

Martínez, I. \& Burgaz, A. R. 2008. El género Cladonia en la Península Ibérica. Supergrupo Crustaceae. Botanica Complutensis 32: 21-36.

Molina, M. C., Divakar, P. K., Millanes, A. M., Sánchez, E., Del-Prado, R., Hawksworth, D. L. \& Crespo, A. 2011. Parmelia sulcata (Ascomycota: Parmeliaceae), a sympatric monophyletic species complex. Lichenologist 43: 585-601. https://doi.org/10.1017/S0024282911000521

Muñiz, D. \& Hladun, N. L. 2011. Flora Liquenológica Ibérica 7 - Calicioides. Sociedad Española de Liquenología, Barcelona.

Muñiz, D., Hladun, N. L. \& Llimona, X. 2009. Mycocalicium llimonae Hladun \& Muñiz (Ascomycota, Eurotiomycetes, Mycocaliciales). Un fong mediterrani tan freqüent con difícil de veure. Ecologia i distribució a Catalunya. Revista Catalana de Micologia 31: 43-48.

Myllys, L., Velmala, S., Holien, H., Halonen, P., Wang, L. S. \& Goward, T. 2011. Phylogeny of the genus Bryoria. Lichenologist 45: 617-638. https://doi.org/10.1017/ S0024282911000132

Myllys, L., Velmala, S., Lindgren, H., Glavich, D., Carlberg, T., Wang, L. S. \& Goward, T. 2014. Taxonomic delimitation of the genera Bryoria and Sulcaria, with a new combination Sulcaria spiralifera introduced. Lichenologist 46: 737-752. https://doi.org/10.1017/S0024282914000346

Nascimbene, J., Nimis, P. L. \& Ravera, S. 2013. Evaluating the conservation status of epiphytic lichens of Italy: A red list. Plant Biosystems 147: 898-904. https://doi.org/10.1080/11 263504.2012.748101

Navarro-Rosinés, P. 1992. Els liquens i els fongs liquenícoles dels substrats carbonatats de Catalunya meridional. Tesis Doctoral, Universitat de Barcelona, Barcelona.

Navarro-Rosinés, P., Calatayud, V. \& Hafellner, J. 2009. Contributions to a revision of the genus Cercidospora (Dothideales) 1. Species on Megasporaceae. Mycotaxon 110: 5-25. https://doi.org/10.5248/110.5

Navarro-Rosinés, P. \& Hafellner, J. 1996. Lichenostigma elongata spec. nov. (Dothideales), a lichenicolous ascomycete on Lobothallia and Aspicilia species. Mycotaxon 57: 211225 .
Navarro-Rosinés, P. \& Roux, C. 1994. Caloplaca cirrochroa specio ofta sed nur supraje konata. Priskribo de la apotecihavaj taloj. Bulletin de la Société Linnéenne de Provence 45: 327-341.

Nimis, P. L. 2016. The lichens of Italy. A second annotated catalogue. EUT, Trieste.

Nimis, P. L., Hafellner, J., Roux, C., Clerc, Ph., Mayrhofer, H., Martellos, S. \& Bilovitz, P. O. 2018. The lichens of the Alps — an annotated checklist. MycoKeys 31: 1-634. https://doi. org/10.3897/mycokeys.31.23568

Oran, S., Özyiğitoğlu, G., \& Öztürk, Ș. 2018. Lichenized and lichenicolous fungi records from Kazdağ 1 (Balıkesir, Turkey). Mantar Dergisi/The Journal of Fungus 9: 39-49.

Orange, A. 2012. Semi-cryptic marine species of Hydropunctaria (Verrucariaceae, lichenized Ascomycota) from north-west Europe. Lichenologist, 44: 299-320. https://doi. org/10.1017/S0024282911000867

Orange, A. 2013. British and other pyrenocarpous lichens. National Museum of Wales, Cardiff. https://museum.wales/ media/13849/Orange-A-2013-British-and-other-pyrenocarpous-lichens.pdf

Otte, V., Yakovchenko, L., Clerc, Ph. \& Westberg, M. 2013. Candelariella commutata sp. nov. for C. unilocularis auct. Medioeur. - an arctic-alpine lichen on calcareous substrata from the Caucasus and Europe. Herzogia 26: 217-222. https://doi.org/10.13158/heia.26.2.2013.217

Paz-Bermúdez, G., López de Silanes, M. E. \& Carballal, R. 1999. Líquenes saxícolas de la Ría de Ribadeo (NO de España): Flora y zonación. Nova Acta Científica Compostelana-Bioloxía 9: 33-46.

Paz-Bermúdez, G., López de Silanes, M. E. \& Carballal, R. 2000. Líquenes saxícolas y terrícolas y hongos liquenícolas interesantes de la costa de Galicia (NW España). Candollea 55: $137-152$.

Paz-Bermúdez, G., López de Silanes, M. E. \& Carballal, R. 2005. The lichen genera Collemopsidium Nyl. and Verrucaria Schrader from the Galician seashores (NW Spain). Nova Hedwigia 80: 73-87. https://doi.org/10.1127/00295035/2005/0080-0073

Pérez-Ortega, S. 2007. Contribución al conocimiento de los líquenes y hongos liquenícolas de Castilla y León, I. Botanica Complutensis 31: 13-22.

Pérez-Ortega, S. \& Álvarez-Lafuente, A. 2006a. Primer catálogo de líquenes y hongos liquenícolas de la Comunidad Autónoma de Cantabria. Botanica Complutensis 30: 5-16.

Pérez-Ortega, S. \& Álvarez-Lafuente, A. 2006b. Primer catálogo de líquenes y hongos liquenícolas de la Comunidad Autónoma de Castilla y León (España). Botanica Complutensis 30: 17-52.

Pino-Bodas, R., Ahti, T., Stenroos, S., Martín, M. P. \& Burgaz, A. R. 2012. Cladonia conista and C. humilis (Cladoniaceae) are different species. Bibliotheca Lichenologica 108: 161-176.

Pino-Bodas, R., Ahti, T., Stenroos, S., Martín, M. P. \& Burgaz, A. R. 2013. Multilocus approach to species recognition in the Cladonia humilis complex (Cladoniaceae, Ascomycota). American Journal of Botany 100: 664-678. https://doi. org/10.3732/ajb.1200162

Poelt, J. \& Hinteregger, E. 1993. Beiträge zur Kenntnis der Flechtenflora des Himalaya.VII. Die Gattungen Caloplaca, Fulgensia und Caloplaca. Bibliotheca Lichenologica 50: $1-247$.

Prieto, M., Aragón, G. \& Martínez, I. 2010a. The genus Catapyrenium s. lat. (Verrucariaceae) in the Iberian Peninsula 
and the Balearic Islands. Lichenologist 42: 637-684. https:// doi.org/10.1017/S0024282910000319

Prieto, M., Martínez, I. \& Aragón, G. 2010b. The genus Placidiopsis in the Iberian Peninsula and the Balearic Islands. Mycotaxon 114: 463-472. https://doi.org/10.5248/114.463

Reese Næsborg, R. 2008. Taxonomic revision of the Lecania cyrtella group based on molecular and morphological evidence. Mycologia 100: 397-416. https://doi.org/10.3852/07-080R

Renobales, G. 1996. Contribución al conocimiento de los líquenes calcícolas del occidente de Vizcaya y parte oriental de Cantabria (N-España). Guineana 2: 1-310.

Renobales, G. \& Noya, R. 1991. Estudio morfológico comparado de Verrucaria maura y V. amphibia en la costa vasca. Acta Botanica Malacitana 16: 149-156.

Renobales, G. \& Noya, R. 1993. Zonation of lichens on a calcareous rocky shore. Nova Hedwigia 57: 489-502.

Rico, V. J., Calatayud, V. \& Giralt, M. 2003. Buellia tesserata and Dimelaena radiata, two closely related species. Lichenologist 35: 117-124. https://doi.org/10.1016/S00242829(03)00012-4

Roux, C. 1978. Complément a l'étude écologique et phytosociologique des peuplements lichéniques saxicoles-calcicoles du SE de la France. Bulletin du Musée d'Histoire Naturelle de Marseille 38: 65-186.

Roux, C., Bültmann, H. \& Navarro-Rosinés, P. 2009. Syntaxonomie des associations de lichens saxicoles-calcicoles du sud-est de la France 1. Clauzadeetea immersae, Verrucarietea nigrescentis, Incertae saedis. Bulletin de la Société Linnéenne de Provence 60: 151-175.

Roux, C., Monnat, J.-Y., Gonnet, D. et al. 2017. Catalogue des lichens et champignons lichénicoles de France métropolitaine (2e éd.). Association Française de Lichénologie, Fontainebleau.

Santesson, R., Moberg, R., Nordin, A., Tønsberg, T. \& Vitikainen, O. 2004. Lichen-forming and lichenicolous fungi of Fennoscandia. Svenska Botaniska Föreningen, Uppsala.

Sarrión, F., Aragón, G. \& Burgaz, A. R. 1999. Studies on mazaediate lichens and calicioid fungi of the Iberian Peninsula. Mycotaxon 71: 169-198.

Sarrión, F. J., Martínez, I. \& Burgaz, A. R. 1993. Líquenes epífitos de Sierra Madrona (Ciudad Real, España). Cryptogamie, Bryologie et Lichénologie 14: 389-400.

Seaward, M. R. D. \& Sipman, H. J. M. 2006. An updated checklist of lichenized and lichenicolous fungi for Egypt. Willdenowia 36: 537-555. https://doi.org/10.3372/wi.36.36151

Seaward, M. R. D., Sipman, H. J. M., Schultz, M., Maassoumi, A. A., Haji Moniri Anbaran, M. \& Sohrabi, M. 2004. A preliminary lichen checklist for Iran. Willdenowia 34: 543-576. https://doi.org/10.3372/wi.34.34218
Shivarov, V. V. \& Stoykov, D. Y. 2012. New records of pyrenocarpous lichenized fungi from Bulgaria. Mycotaxon 121: 123-138. https://doi.org/10.5248/121.133

Smith, C. W., Aptroot, A., Coppins, B. J., Fletcher, A., Gilbert, O. L., James, P. W. \& Wolseley, P. A. (Eds.) 2009. The lichens of Great Britain and Ireland (2nd ed.). The British Lichen Society, London.

Spribille, T., Thor, G., Bunnell, F. L., Goward, T. \& Björk, C. R. 2008. Lichens on dead wood: species-substrate relationships in the epiphytic lichen floras of the Pacific Northwest and Fennoscandia. Ecography 31: 741-750. https://doi. org/10.1111/j.1600-0587.2008.05503.x

Svensson, M., Ekman, S., Klepsland, J. T. et al. 2017. Taxonomic novelties and new records of Fennoscandian crustose lichens. MycoKeys 25: 51-86. https://doi.org/10.3897/ mycokeys. 25.13375

Tarasova, V. N., Obabko, R. P., Himelbrant, D. E., Boychuk, M. A., Stepanchikova, I. S. \& Borovichev, E. A. 2017. Diversity and distribution of epiphytic lichens and bryophytes on aspen (Populus tremula) in the middle boreal forests of Republic of Karelia (Russia). Folia Cryptogamica Estonica 54: 125-141. http://dx.doi.org/10.12697/fce.2017.54.16

Tehler, A., Ertz, D. \& Irestedt, M. 2013. The genus Dirina (Roccellaceae, Arthoniales) revisited. Lichenologist 45: 427476. https://doi.org/10.1017/S0024282913000121

Terrón, A., Burgaz, A. R. \& Álvarez-Andrés, J. 2000. Líquenes de la provincia de Zamora (España). Botanica Complutensis 24:9-43.

Tönsberg, T. 1992. The sorediate and isidiate, corticolous, crustose lichens in Norway (Sommerfeltia, 14). Botanical Garden and Museum, University of Oslo, Oslo.

Tretiach, M. \& Hafellner, J. 1998. A new species of Catillaria from coastal Mediterranean Regions. Lichenologist 30: 221-229. https://doi.org/10.1006/lich.1998.0126

Velmala, S., Myllys, L., Goward, T., Holien, H. \& Halonen, P. 2014. Taxonomy of Bryoria section Implexae (Parmeliaceae, Lecanorales) in North America and Europe, based on chemical, morphological and molecular data. Annales Botanici Fennici 51: 345-371. https://doi.org/10.5735/085.051.0601

Woods, R. G. \& Coppins, B. J. 2012. A conservation evaluation of British lichens and lichenicolous fungi. Species Status 13. Joint Nature Conservation Committee, Peterborough.

Yazıcı, K., Aptroot, A., Aslan, A., Etayo, J., Spier, L. \& Karagöz, Y. 2010. Lichenized and lichenicolous fungi from nine different areas in Turkey. Mycotaxon 111: 113-116. https:// doi.org/10.5248/111.113

Zhdanov, I. 2010. New and rare lichen records from the Central Siberian Biosphere Reserve (Krasnoyarsk Krai, Russia). Folia Cryptogamica Estonica 47: 101-104. 